EPJ Web of Conferences 66, 07011 (2014)

DOI: $10.1051 /$ epjconf/ 20146607011

(C) Owned by the authors, published by EDP Sciences, 2014

\title{
Electron Capture and Beta-Decay Rates for the Collapse of $\mathrm{O}+\mathrm{Ne}+\mathrm{Mg}$ Cores
}

\author{
Yi Hua Lam ${ }^{1, a}$, Gabriel Martínez-Pinedo ${ }^{1,2}$, Karlheinz Langanke ${ }^{2,1}$, Samuel Jones ${ }^{3}$, Raphael \\ Hirschi $^{3}$, Remco G. T. Zegers ${ }^{4,5}$, and B. Alex Brown ${ }^{4,5}$ \\ ${ }^{1}$ Institut für Kernphysik, Technische Universität Darmstadt, 64289 Darmstadt, Germany \\ ${ }^{2}$ GSI Helmholtzzentrum für Schwerionenforschung, Planckstraße 1, 64291 Darmstadt, Germany \\ ${ }^{3}$ Astrophysics Group, Lennard Jones Building, Keele University ST5 5BG, United Kingdom \\ ${ }^{4}$ NSCL, Michigan State University, East Lansing, Michigan 48824-1321, USA \\ ${ }^{5}$ Dept. of Phys. and Astro., Michigan State University, East Lansing, Michigan 48824, USA
}

\begin{abstract}
We present a new set of electron $\left(e^{-}\right)$capture and $\beta$-decay rates that improves previous calculations by Takahara et al. and Oda et al. by: (a) incorporation of recent charge-exchange and $\beta$-decay data, (b) contributions of forbidden transitions, and (c) inclusion of $e^{-}$screening corrections. The experimental nuclear input is supplemented by theoretical data based on large-scale shell model calculations in the full $s d$-shell space using the USDB interaction. The impact of these new rates on the late-stage stellar evolution of $8.8 M_{\odot}$ star is demonstrated.
\end{abstract}

\section{Introduction}

Stars in the mass range of $8 \lesssim M / M_{\odot} \lesssim 12$ evolve to degenerate $\mathrm{O}+\mathrm{Ne}$ or $\mathrm{O}+\mathrm{Ne}+\mathrm{Mg}$ cores after $\sim 10^{6}$ years [1]. In such a very abundant ${ }^{20} \mathrm{Ne}$ and ${ }^{24} \mathrm{Mg}$ matter composition, the $e^{-}$captures on these two nuclear species play the pivotal role in reducing the $e^{-}$pressure support, which is supplied by the degenerate relativistic $e^{-}$gas to against the gravitational contraction of the stellar core. Under the situation of loosing $e^{-}$to capture process, these stars are driven toward the core collapse stage. Besides, the core environment is cooled by the produced neutrinos $(v)$ which carry away energy and leave the star unhindered as long as the density is less than $\sim 10^{11} \mathrm{~g} / \mathrm{cm}^{3}$. Moreover, the $e^{-}$capture process transmute the stellar composition to be more neutron rich by changing protons in the nucleus to neutrons. Therefore, stars in this mass range are crucial in nucleosynthesis of certain nuclides [2].

The $e^{-}$-capture supernovae and late-stage stellar evolution simulation of $8-12 M_{\odot}$ stars utilize the $e^{-}$-capture rates of ${ }^{20} \mathrm{Ne}$ and ${ }^{24} \mathrm{Mg}$, and $\beta$-decay rates of their daughters, i.e. ${ }^{20} \mathrm{~F}$ and ${ }^{24} \mathrm{Na}$, respectively, from Ref. [3]. However, these rates were tabulated in a sparse density-temperature $(\rho-T)$ grid. This may hide physics of critical $\rho$ - $T$ region from being manifested in simulations [4]. Nevertheless, rates of Oda et al. [3] and Takahara et al. [5] had been calculated prior to the charge-exchange experiments of Gamow-Teller (GT) strength distributions for ${ }^{20} \mathrm{Ne}$ and ${ }^{24} \mathrm{Mg}$ [6]. In present work, we improve the $e^{-}$-capture and $\beta$-decay rates by including recently measured GT transitions and also forbidden transition, which are the main contributions to the rates, and by considering Coulomb screening effects in dense stellar environment.

ae-mail: LamYiHua@gmail.com

This is an Open Access article distributed under the terms of the Creative Commons Attribution License 2.0, which permits unrestricted use, distribution, and reproduction in any medium, provided the original work is properly cited. 


\section{EPJ Web of Conferences}

\section{Formalism}

The $e^{-}$of $8-12 M_{\odot}$ stars in the late-stage stellar environment form a degenerate relativistic Fermi gas under temperatures, $T=10^{8}-10^{10} \mathrm{~K}$ and densities, $\rho=10^{8}-10^{10} \mathrm{~g} / \mathrm{cm}^{3}$. The total rate of $e^{-}$ capture or $\beta^{-}$decay is stated as [7],

$$
\lambda^{\alpha}=\frac{\ln 2}{K} \sum_{i} \frac{\left(2 J_{i}+1\right) e^{-E_{i} /(k T)}}{G(Z, A, T)} \sum_{j} B_{i j} \Phi_{i j}^{\alpha},
$$

where the superscript $\alpha$ is for either $e^{-}$capture (ec) or $\beta^{-}$-decay $\left(\beta^{-}\right)$, and the sums in $i$ and $j$ run over states in the parent and daughter nuclei, respectively. The constant $K=6143.6 \pm 1.7 \mathrm{~s}$, is quoted from superallowed Fermi transitions [8], the partition function of the parent nucleus, $G(Z, A, T)=$ $\sum_{i}\left(2 J_{i}+1\right) \exp \left[-E_{i} /(k T)\right]$, and the reduced transition probability of the nuclear transition is [9],

$$
B_{i j}(G T)=\left(\frac{g_{A}}{g_{V}}\right)_{\text {eff. }}^{2} \frac{\left|\left\langle j\left\|\sum_{k} \sigma^{k} t_{ \pm}^{k}\right\| i\right\rangle\right|^{2}}{2 J_{i}+1}, \quad \text { and }\left(\frac{g_{A}}{g_{V}}\right)_{\text {eff. }}^{2}=0.77\left(\frac{g_{A}}{g_{V}}\right)_{\text {bare }}^{2} .
$$

Each of the phase space integral for $e c$ and $\beta^{-}$in Eq. (2) is calculated by

$$
\begin{aligned}
\Phi_{i j}^{e c} & =\int_{w_{l}}^{\infty} w p\left(Q_{i j}+w\right)^{3} F(Z, w) \times S_{e}(w) d w, \text { and } \\
\Phi_{i j}^{\beta^{-}} & =\int_{1}^{Q_{i j}} w p\left(Q_{i j}-w\right)^{3} F(Z+1, w) \times\left[1-S_{e}(w)\right] d w,
\end{aligned}
$$

respectively, where the $w$ is the total, rest mass and kinetic, energy of the $e^{-}, Q_{i j}$ is the total energy available in $\beta$ decay, $p=\sqrt{w^{2}-1}$ is the momentum, and $S_{e}$ is the Fermi-Dirac distribution of $e^{-}$. Both rates of ground state (g.s.) transition and excited-state transition can be written as [7]

$$
\lambda_{i f}=\frac{\ln 2 B_{i f}}{K}\left(\frac{k T}{m_{e} c^{2}}\right)^{5}\left(2 J_{i}+1\right) \zeta\left[F_{4}(\eta)+2 \chi F_{3}(\eta)+\chi^{2} F_{2}(\eta)\right],
$$

where $\chi=Q_{i f} /(k T), \eta=\left(\mu_{e}-Q_{i f}\right) /(k T), Q_{i f}$ is the $Q$-value for the respective transition, $\mu_{e}$ is the chemical potential, and $F_{k}$ are the relativistic Fermi integrals of order $k$. The Boltzmann probability, $\zeta=\exp \left[-E_{i} /(k T)\right]$, is equal to unity for g.s. transition, and $E_{i}$ is the respective excitation energy.

\section{Results}

We obtained theoretical GT strengths within the shell-model approach in the full $s d$ shell using USDB interaction [10]. However, for the relevant $\rho$-T range, both $e^{-}$-capture and $\beta$-decay rates of ${ }^{20} \mathrm{Ne} \rightleftharpoons{ }^{20} \mathrm{~F}$ and ${ }^{24} \mathrm{Mg} \rightleftharpoons{ }^{24} \mathrm{Na}$ are fully determined by the experimental GT strengths [6]. Rates shown in Figs. 1-4 were calculated without Coulomb screening effect. This medium effect was included in the final rates.

Rates of Urca pairs ${ }^{20} \mathbf{N e} \rightleftharpoons{ }^{20} \mathbf{F}$. Comparing to previous calculations of Refs. [3, 5], c.f. Fig. 1, we find that the $e^{-}$capture on ${ }^{20} \mathrm{Ne}$ is enhanced by several orders of magnitude, in the density range $4 \lesssim \rho /\left(10^{9} \mathrm{~g} / \mathrm{cm}^{3}\right) \lesssim 10$ and temperatures below $0.7 \mathrm{GK}$, due to the contribution of the second forbidden transition from ${ }^{20} \mathrm{Ne}$ g.s. to ${ }^{20} \mathrm{~F}$ g.s. The forbidden transition also enhances $\beta$-decay rates in this density regime, c.f. Fig. 2 . The $e^{-}$capture rate of $\left({ }^{20} \mathrm{Ne}\right) 2_{1}^{+} \rightarrow\left({ }^{20} \mathrm{~F}\right) 2^{+}$is higher than the rate of ${ }^{20} \mathrm{Ne}$ g.s. to ${ }^{20} \mathrm{~F}$ g.s. (double-dot-dashed line) at density $\rho \lesssim 9.3 \times 10^{9} \mathrm{~g} / \mathrm{cm}^{3}$, c.f. Fig. 1 . The reason is, the $\mu_{e}$ is much lower than the $e^{-}$-capture $Q$-value in the early evolution stage of an O-Ne$\mathrm{Mg}$ core, and the $Q_{i f}$ is decreased with increasing $E_{i}$. Hence, the decrement of rate caused by the Boltzmann probability, $\exp \left[-E_{i} /(k T)\right]$, for the thermal population of the excited state with increasing $E_{i}$ in Eq.(4) is compensated by the exponential increment, i.e. higher value of Fermi integrals in Eq.(4), of the number of $e^{-}$contributing to the capture process. 


\section{INPC 2013}

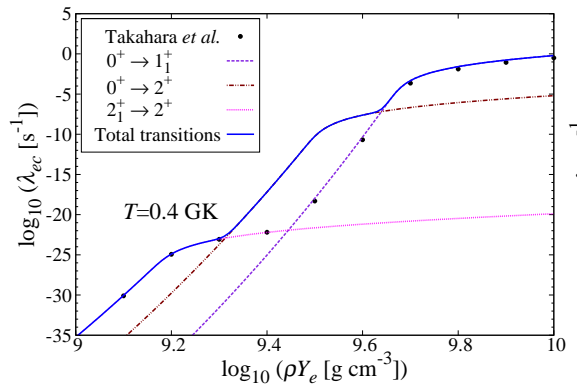

Fig. 1. The $e^{-}$capture rates of ${ }^{20} \mathrm{Ne}$.

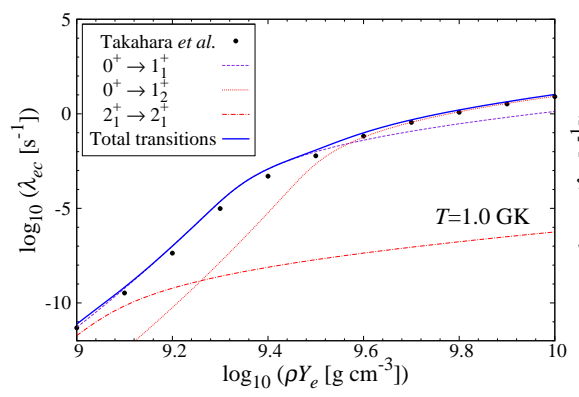

Fig. 3. The $e^{-}$capture rates of ${ }^{24} \mathrm{Mg}$.

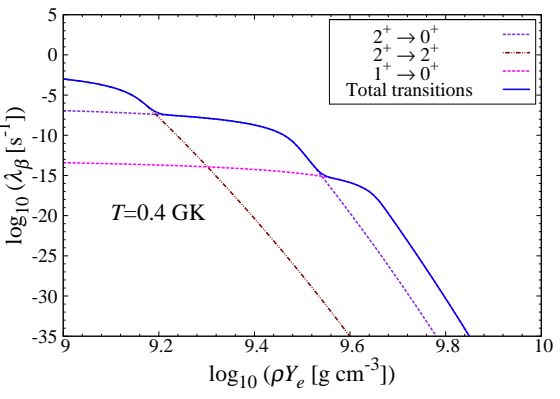

Fig. 2. The $\beta^{-}$decay rates of ${ }^{20} \mathrm{~F}$.

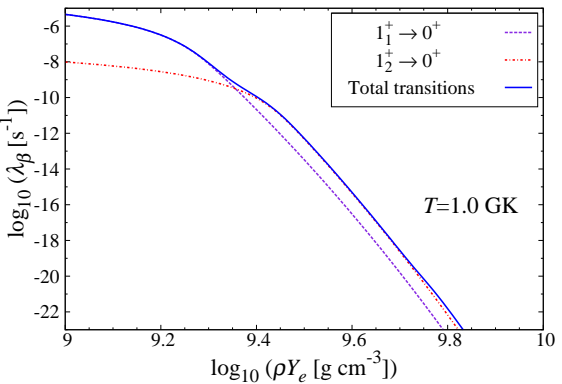

Fig. 4. The $\beta^{-}$decay rates of ${ }^{24} \mathrm{Na}$.

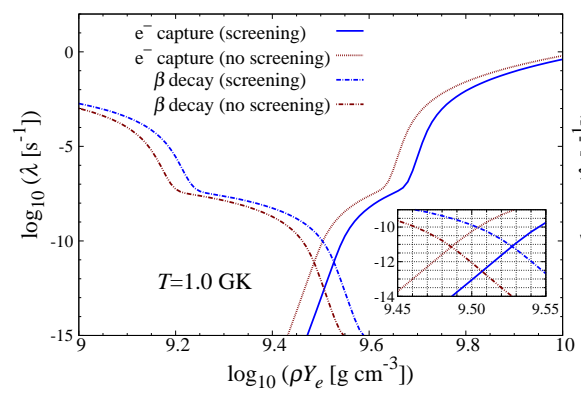

Fig. 5. The Urca pair of ${ }^{20} \mathrm{Ne} \rightleftharpoons{ }^{20} \mathrm{~F}$.

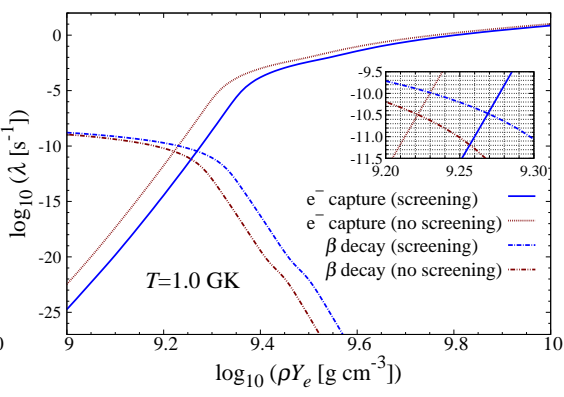

Fig. 6. The Urca pair of ${ }^{24} \mathrm{Mg} \rightleftharpoons{ }^{24} \mathrm{Na}$.

Rates of Urca pairs ${ }^{24} \mathbf{M g} \rightleftharpoons{ }^{24} \mathbf{N a}$. Overall, due to the recent $\beta$-decay data from Nishimura et al. [6], the $e^{-}$capture on ${ }^{24} \mathrm{Mg}$ is enhanced by about a factor of two compared to Takahara et al. [5], c.f. Fig. 3. The $e^{-}$capture on ${ }^{24} \mathrm{Mg}$ dominates over $\beta$ decay of ${ }^{24} \mathrm{Na}$ at $\rho \gtrsim 9.3 \times 10^{9} \mathrm{~g} / \mathrm{cm}^{3}$, c.f. Fig. 6 . Although forbidden transitions do not contribute to the $e^{-}$capture rate, the $\beta$ decay rate of ${ }^{24} \mathrm{Na}$ is enhanced by these transitions at temperature $T \lesssim 0.4 \mathrm{GK}$.

Coulomb screening effect. We included this effect according to Ref. [11]. Coulomb screening reduces $e^{-}$capture rate in dense astrophysical environment in two ways. First, the threshold energy in the medium is increased, $Q_{i f}^{e c, \text { med }}=Q_{i f}+\Delta Q_{c}(Z)$. Second, the chemical potential of $e^{-}$is decreased, $\mu_{e}^{\text {med }}=\mu_{e}-V_{s}$. Contrarily, $\beta$-decay rate is increased in two ways. First, the threshold energy in the medium is decreased, $Q_{i f}^{\beta, \text { med }}=Q_{i f}-\Delta Q_{c}(Z+1)$. Second, the decrement of $\mu_{e}$ causes the reduction of Pauli blocking in the final state, i.e. lower values of $\left[1-S_{e}(w)\right]$ in Eq.(3b). The comparison of rates with screening effect and without screening effect is shown in Figs. 5 and 6. 
Stellar evolution simulation. Jones et al. showed that stellar evolution simulations using recently updated rates produce lower temperatures at the start of $e^{-}$-capture phase for super-AGB star progenitors [4]. Here, we performed a stellar evolution simulation of $8.8 M_{\odot}$ star, which is failed massive star (see Ref. [4] for definition). The central $\rho$ - $T$ of the late-stage region is shown in Fig. 7. The first dip at the central density, $\rho_{c} \approx 9.1 \times 10^{9} \mathrm{~g} / \mathrm{cm}^{3}$ is due to ${ }^{25} \mathrm{Mg} \rightleftharpoons{ }^{25} \mathrm{Al}$, whereas the second dip at $\sim 9.2 \times 10^{9} \mathrm{~g} / \mathrm{cm}^{3}$ is due to ${ }^{23} \mathrm{Na} \rightleftharpoons{ }^{23} \mathrm{Ne}$. Both dips correspond to the URCA cooling, when $\beta$ decay and $e^{-}$capture proceed on similar timescale and thus provide neutrino cooling. The near-adiabatic contraction is noticeable at $9.2 \lesssim \rho_{c} /\left(10^{9} \mathrm{~g} / \mathrm{cm}^{3}\right) \lesssim 9.5$. During the contraction, there are two late $\mathrm{Ne}-\mathrm{O}$ shell flashes which provide an additional support for the outer layers of the core, hence the center expands (lower down the density) and cools adiabatically each time before contracting again. The heat releasing process of ${ }^{24} \mathrm{Mg} \rightarrow{ }^{24} \mathrm{Na} \rightarrow{ }^{24} \mathrm{Ne}$ only happens on the input rates of Takahara et al. [5] (in a coarse $\rho$ - $T$ grid) at the region of $9.55 \lesssim \rho_{c} /\left(10^{9} \mathrm{~g} / \mathrm{cm}^{3}\right) \lesssim 9.8$. However, it does not happen on the other two sets of fine grid, because consistent resolution of $e^{-}$capture and $\beta$-decay rates are used in the simulation. Interestingly, these two input rates cause a few spikes at $\rho_{c} \approx 9.85 \times 10^{9} \mathrm{~g} / \mathrm{cm}^{3}$. It may be the center heats up quickly when the $e^{-}$capture on ${ }^{20} \mathrm{Ne}$ starts, but at some point it becomes convectively unstable because there is so much energy being released. This unstable behavior is more profound for the present input rates.

\section{Conclusion}

We present an improved calculation of $e^{-}$ capture and $\beta$-decay rates of ${ }^{20} \mathrm{Ne} \rightleftharpoons{ }^{20} \mathrm{~F}$ and ${ }^{24} \mathrm{Mg} \rightleftharpoons{ }^{24} \mathrm{Na}$. The impact of new rates on latestage stellar evolution of $8.8 M_{\odot}$ model at core density $\rho_{c} \gtrsim 9.85 \times 10^{9} \mathrm{~g} / \mathrm{cm}^{3}$ is demonstrated. Rates of above Urca pairs and other Urca pairs are available upon request from the author.

\section{References}

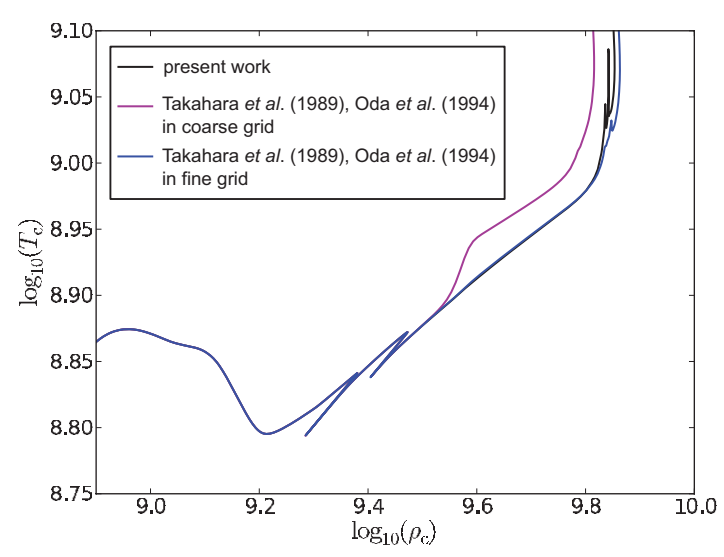

[1] K. Nomoto, Astrophys. J. 277, 791 (1984).

[2] H. Ning et al., Astrophys. J. 667, L159 (2007); R.D. Hoffman et al., Astrophys. J. 667, L159 (2007); S. Wanajo et al., Astrophys. J. 695, 208 (2009).

[3] T. Oda et al., At. Data Nucl. Data. Tables 56, 231 (1994).

[4] S. Jones et al., Astrophys. J. 772, 1 (2013).

[5] M. Takahara et al., Nucl. Phys. A 504, 167 (1989).

[6] B.D. Anderson et al., Phys. Rev. C 43, 50 (1991); S. Rakers et al., Phys. Rev. C 65, 044323 (2002); R.G.T. Zegers et al., Phys. Rev. C 78, 014314 (2008); D. Nishimura et al., Eur. J. Phys. A 47, 155 (2011).

[7] G.M. Fuller, W.A. Fowler, and M.J. Newman, Astrophys. J. Supp. 42, 447 (1980); Astrophys. J. Supp. 48, 279 (1982); Astrophys. J. 293, 1 (1985).

[8] I.S. Towner and J.C. Hardy, Rep. Prog. Phys. 73, 046301 (2010).

[9] K. Langanke and G. Martínez-Pinedo, At. Data Nucl. Data. Tables 79, 1 (2001).

[10] W.A. Richter and B.A. Brown, Phys. Rev. C 74, 034315 (2006).

[11] A. Juodagalvis et al., Nucl. Phys. A 848, 454 (2010). 